\title{
ON THE FORMS OF CURVES PRESENTED BY THE MICHELSON INTERFEROMETER.
}

By John Cutler Shedd.

\section{Preliminary.}

THE theory of the interference phenomena produced by this instrument has been entered into somewhat in detail by Professor A. A. Michelson. ${ }^{1}$ In the present paper it is proposed, first to follow the ground plan presented by Professor Michelson and then to give a point of view which is believed to be novel.

\section{PART I.}

In Fig. I is given a plan view of the usual form of interferometer.

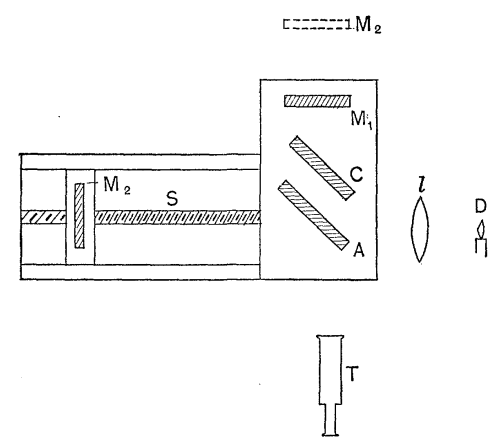

Fig. 1.

$M_{1} M_{2} \equiv$ heavily silvered mirrors.

$A \equiv$ plate, half silvered on side toward $M_{1}$.

$C \equiv$ plate of same thickness as $A$ and placed parallel to it. Plate $C$ is called the compensator.

$M_{2}$ is on a movable carriage controlled by screw $S$.

The interference pattern is viewed by the eye, or telescope, placed at $T$.

${ }^{1}$ Phil. Mag. [5], I3, 1882, pp. 236-242. 
It is evident that the mirror $M_{2}$ may be regarded as occupying a position behind $M_{1}$, as shown in the dotted lines. Regarding $M_{2}$ as so placed, we may, as has been shown by Professor Michelson, suppress everything excepting $M_{1}$ and $M_{2}$.

Let $d e$ be drawn parallel to the line of intersection of the planes of $M_{1}$ and $M_{2}$ and let the angle of intersection be $\varphi$.

The whole phenomenon may now be discussed on the basis of a source of light $S$ placed in the plane of $M_{1}$ and of its image in $M_{2}$.

In this discussion the following notation will be used:

$t_{0} \equiv$ Perpendicular distance between $M_{1}$ and $M_{2}$. On the instrument this is controlled by the screw $S$ (Fig. I).

$\varphi \equiv$ Angle between $M_{1}$ and $M_{2}$. This is controlled by adjusting screws at the back of $M_{1}$.

$P \equiv$ Perpendicular distance from $M_{1}$ to the plane containing the interference pattern. This plane of maximum distinctness is called the focal plane.

$\theta \equiv$ Angle subtended by the source of light at point $P$ on the focal plane, in a plane perpendicular to the plane of $\varphi$.

$i \equiv$ Same in plane parallel to plane of $\varphi$.

$\Delta \equiv$ Difference of path traversed by the two rays producing interference at $P$.

Professor Michelson has shown that the value of $\Delta$ is given by the equation

$$
\Delta=2 \frac{t_{0}+P \tan i \tan \varphi}{\sqrt{\mathrm{I}+\tan ^{2} i+\tan ^{2} \varphi}} .
$$

In general $\Delta$ has all values but the value which will give the most distinct fringes is given by the conditions

and

$$
\frac{\partial \Delta}{\partial \theta}=0
$$

$$
\frac{\partial \Delta}{\partial i}=0 \text {. }
$$

This gives the value of $P$ for which the phenomenon of interference is most distinct. The value is

$$
P=\frac{t_{0}}{\tan \varphi} \tan i
$$


It follows from equation (2) that if $t_{0}=0$ or $i=0$ then $P=0$ and the interference fringes are on the surface of $M_{1}$. If $t_{0}=0$ and $\varphi=0$, or $i=0$ and $\varphi=0$ then $P$ is indeterminate. If $\varphi=0$ then $t_{0}$ is everywhere uniform and $P=\infty$. If $\varphi$ and $i$ have same sign $P$ is positive and the fringes are in front of $M_{1}$. If $\varphi$ and $i$ have opposite signs $P$ is negative and the fringes are behind $M_{1}$.

If $M_{2}$ is behind $M_{1}$, then $\varphi$ is regarded as positive and $t_{0}$ as positive. If $M_{2}$ is in front of $M_{1} \varphi$ and $t_{0}$ are both regarded as negative. The plane containing the interference pattern is called the Focal plane, and upon this plane the eye or telescope must be focused in order to get the most distinct view of the fringes. The Focal plane is parallel to the plane of $M_{1}$ and at a distance $P$ from it.

Any point on the Focal plane is determined by the equations

$$
\left.\begin{array}{l}
x=P \tan i \\
y=P \tan \theta
\end{array}\right\}
$$

and the form of curve produced in the interference pattern is found by substituting equations (3) in equation (I). This gives the following equation of the interference curves:

$$
\Delta^{2} y^{2}=\left(4 P^{2} \tan ^{2} \varphi-\Delta^{2}\right) x^{2}+8 t_{0} P^{2} \tan \varphi x+P^{2}\left(4 t_{0}^{2}-\Delta^{2}\right) .
$$

The further discussion of the subject is an analysis of equation (4), and it is very essential to its good understanding that every term involved be thoroughly apprehended. We therefore repeat:

$2 t_{0} \equiv$ Perpendicular distance between $M_{1}$ and its image in $M_{2}$, optically considered [this reduces to the value of $\Delta$ when $i$ and $\theta$ both are zero].

$\varphi \equiv$ angle that $M_{1} M_{2}$ make with each other [physically the angle by which they differ from $90^{\circ}$ ].

$P \equiv$ Perpendicular distance of the focal plane from $M_{1}$.

$i, \theta \equiv$ Angles of incidence upon the focal plane, of the interfering beams : $i$, being measured in the plane parallel to and $\theta$ in the plane normal to the plane of $\varphi$. The focal plane is normal both to the plane of $i$ and of $\theta$.

$\Delta=$ Difference of path of the interfering beams.

In the article already referred to Professor Michelson expresses equation (4) in the equivalent form 
No. 5.]

$$
\Delta^{2} y^{2}=\left(4 S^{2} K^{2}-\Delta^{2}\right) x^{2}+8 T S K D x+\left(4 T^{2}-\Delta^{2}\right) D^{2}
$$

and then points out what curves are represented by equation (5) and the values of the constants necessary in each case.

It would seem to be equally as simple and more satisfactory to refer directly to equation (4). The following are the principal cases that arise.

(I) $\Delta=0$; Equation (4) becomes

$$
x=\frac{t_{0}}{\tan \varphi}
$$

representing a straight line.

(2) $\varphi=0$; then

$$
x^{2}+y^{2}=\frac{P^{2}}{\Delta^{2}}\left(4 t_{0}^{2}-\Delta^{2}\right)
$$

giving circular fringes.

(3) $\Delta=2 P \tan \varphi$. In this case equation (4) becomes

$$
y^{2}=\frac{4 F t_{0}}{\Delta} x_{1}
$$

In equation (8) this axis of $y$ has been shifted so that

$$
x_{1}=x-P \frac{4 t_{0}^{2}-\Delta^{2}}{4 t_{0} 4}
$$

thus giving the central equation. Equation (8) is that of a parabola

(4) $\Delta>2 P \tan \varphi ;$ Equation (4) takes the form

$$
\frac{x^{2}}{A^{2}}+\frac{y^{2}}{B^{2}}=\mathrm{I}
$$

where

and

$$
A^{2}=\frac{\Delta^{2} P^{2}\left(4 t_{0}^{2}-\Delta^{2}\right)}{\Delta^{2}-4 P^{2} \tan ^{2} \varphi}
$$

$$
B^{2}=P^{2}\left(4 t_{0}{ }^{2}-\Delta^{2}\right)
$$

thus giving the semi-axes of the ellipse

(5) $\Delta<2 P \tan \varphi$. In this case the hyperbola

$$
\frac{x^{2}}{A^{2}}-\frac{y^{2}}{B^{2}}=\mathrm{I}
$$


is obtained where

and

$$
A^{2}=\frac{\Delta^{2} P^{2}\left(\Delta^{2}-4 t_{0}^{2}\right)}{\Delta^{2}-4 P^{2} \tan ^{2} \varphi}
$$

$$
B^{2}=P^{2}\left(\Delta^{2}-4 t_{0}^{2}\right)
$$

A more detailed consideration of these cases is reserved for the second part of the paper.

\section{PART II.}

The curves just considered may also be very readily developed by considering their eccentricity. The general expression for the eccentricity of equation (4) is

$$
e=\sqrt{\mathrm{I}+A / B}
$$

where $A$ is the coefficient of $x^{2}$ and $B$ of $y^{2}$. In the present case this becomes the very simple expression

$$
e=\frac{2 P \tan \varphi}{\Delta}
$$

Also since in equation (2)

we get

$$
P=\frac{t_{0}}{\tan \varphi} \tan i
$$

$$
e=\frac{2 t_{0} \tan i}{S}
$$

Finally substituting the value of $\Delta$ given in equation (I) we get

or

$$
e=\sin i \cos i \sqrt{\mathrm{I}+\tan ^{2} i+\tan ^{2} \theta}
$$

$$
e=\sin i \sqrt{\mathrm{I}+\tan ^{2} \theta} \cos ^{2} i .
$$

The various cases that rise can be readily covered by equations (II) to (14). The following will be considered.

Case $I . \quad \Delta=\mathrm{O}$; then by equation (I I) or (I 2 )

$$
e=\infty
$$

and the curves are straight lines. If, however, $t_{0}$ be also zero, then by equations (II) or (I 2) $e$ is indeterminate. The meaning of this is that mirrors $M_{1}$ and $M_{2}$ coincide and hence there is but a single 
source of light. The conditions therefore are not favorable for interference.

However, if $t_{0}=0$ and $\varphi>0$ then $P=0$ and it is also apparent that both $i$ and $\theta$ are zero. If $i$ and $\theta$ are both zero it is apparent by equation (I3) that $e=0$, and the curves if existent, would be circles. This point will be returned to later.

Case 2. $t_{0}=0$ and $\varphi>0$; then $P=0$, and the central band will be a straight line (corresponding to $\Delta=0$ and $e=\infty$ ) flanked on either side by bands of opposite curvature $(\Delta>0 e<\infty)$. The fringes on one side are behind the mirror ( $P$ negative) while those on the other side are in front of the mirror ( $P$ positive).

Referring to equations (I) and (I I) viz. :

$$
\Delta=2 \frac{t_{o}+P \tan i \tan \varphi}{\sqrt{\mathrm{I}+\tan ^{2} i+\tan \theta}}
$$

and

$$
e=\frac{2 P \tan \varphi}{\Delta}
$$

we see that since $P$ is small, being zero for the central band, the values of $i$ and $\theta$ rapidly increase, hence ( $t_{0}$ also being very small) the value of $\Delta$ is small (Eq. I) and the value of $e$ (Eq. I I) will be very large. The larger the value of $\varphi$ the more rapidly does $i$ change ( $i$ being in the plane of $\varphi$ ) and the more slowly does the value of $e$ fall. Furthermore the central straight band lies parallel to the intersection of $M_{1}$ and $M_{2}$ and hence the more rapidly $i$ changes (i.e., the larger the value of $\varphi$ ) the closer together will the fringes be. Hence as $\varphi$ is made to increase the field becomes covered with narrow fringes that are practically straight lines. If now $M_{2}$ be made to recede, so as to increase $t_{0}$, the fringes soon appear curved. Again increasing the value of $\varphi$ tends to make the fringes straight, but a limit is soon reached where they are too narrow to be distinguishable.

For work over a limited range of $t_{0}$, it is frequently convenient to use straight fringes. It is also apparent that under these conditions $P$ is practically zero and hence the telescope is to be focused on mirror $M$. 
This point is very well illustrated in the work of Morley and Rogers in their research on the thermal expansion of metals. ${ }^{1}$

Case 3. $\varphi=0$. In this case $e=0$ and the fringes are circles. If $\varphi=0$ then $P=\infty$ (Eq. 2) and the telescope must be focused for parallel light. Regarding equation (7) we see that the radius of each circle is

$$
R=\frac{P}{\Delta} \sqrt{\left(2 t_{0}+\Delta\right)\left(2 t_{0}-\Delta\right)}
$$

But $2 t_{0}-\Delta=n \lambda$ and $2 t_{0}+\Delta=2 \Delta$ (approximately), so that

$$
R=P \sqrt{\frac{n \lambda}{t_{0}}} \text { (approximately). }
$$

.$R \propto \sqrt{n}$ showing that the circular fringes follow the same law as do Newton's rings.

Case 4. $\varphi>0$, but small. We obtain by equation (6), if we put $2 t_{0}=\Delta$, and from equation (12)

$$
e=\tan i \text {. }
$$

For small values of $i$ the fringes are circular, becoming elliptical as $i$ increases. Thus for values of $i<5^{\circ}, e<0$. I.

It is easy to so adjust $t_{0}$ and $\varphi$ that circular fringes shall be seen in the center of the field of view, surrounded by increasingly elliptical fringes as $i$ increases in value.

In this connection a very interesting case arises. For large values of $t_{0}, \varphi$ and $i$ being of opposite sign, the focal plain is behind $M_{1}$. Under these conditions $t_{0}$ and $\varphi$ are positive, $i$ and $P$ negative. By equation (2) $\Delta$ is negative and $e$, of course, positive. Also for large values of $t_{0}$ the value of $P$ given by equation (3) is large, and the value of $i$ is necessarily very small, hence $e$ is small (Eq. (I2)), and the fringes are circles. If now $M_{2}$ be advanced until $t_{0}=0$ then $P=0$ (Eq. (2)) and the focal plane is on $M_{1}$, or, more correctly, the focal plane intersects $M_{1}$ in the field of vision. At this point $e=\tan i$ (practically) and the fringes are still circles. Since $t_{0}=0$ at this point circular chromatic fringes may be obtained. These fringes are beautifully distinct and brilliant. 
If $M_{1}$ be still further advanced so as to bring $M_{2}$ in front of $M_{1}$, then $t_{0}$ and $\varphi$ change sign, becoming negative; $i$ and $P$ become positive, while $\Delta$ remains negative. ${ }^{1}$

The value of $\Delta$ then becomes

$$
\Delta=2 \frac{-t_{0}+P \tan i \tan \varphi}{\sqrt{1+\tan ^{2} i+\tan ^{2} \varphi}}
$$

and $t_{0}>P \tan i \tan \varphi$, so that $\Delta$ is negative. As $M_{1}$ is still further advanced $t_{0}$ increases in value and so also $P$. For a time the value of $\Delta$ will diminish and the value of $e$ (Eqs. I I or I 2 ) increase. The result is that the curves rapidly change from circles $(e=0) t_{0}$ ellipses $(e>0$ but $<\mathrm{I})$ then to the parabola $(e=\mathrm{I})$ becoming hyperbolæ $(e>\mathrm{I})$ and finally giving the straight $\operatorname{line}^{2}(e=\infty)$.

If after the minimum value of $\Delta$ is reached $t_{0}$ be still further increased in value, the increased value of $P$ is more than counterbalanced by the diminished value of $\tan i$, so that the term $P \tan i$ $\tan \varphi$ diminishes in value. The value of $\Delta$ therefore increases and the value of $e$ diminishes. The result is that when $M_{2}$ is further advanced the curves change curvature (the conjugate hyperbolæe coming into view) and pass through the hyperbolic, parabolic, elliptic forms and finally again appear as circles.

The foregoing case furnishes a very ready method of finding the chromatic fringes (zero position of the instrument) without any previous acquaintance with the instrument. The scheme is as follows: Having secured fringes with the sodium flame in the usual manner, run $M_{2}$ out 6 or $7 \mathrm{~mm}$. and adjust $M_{2}$ so that the parallax is a minimum. This will give a small value of $\varphi$. Now advance $M_{2}$ through the zero position until the curves are hyperbolæ. Finally reverse the motion and let $M_{2}$ recede until the fringes just begin to become circular. If, now, white light be substituted for the sodium flame, and $M_{2}$ be made to slowly recede by means of the

1 The negative sign before $\Delta$ indicates that the increment of $\Delta$, as $i$ increases, is a diminishing increment. The result is that the focal plane becomes a curved surface concave to the observer for negative values of $P$ ( $P$ diminishing as $i$ increases in value). This can be detected by the eye, the fringes appearing to lie on a concave surface.

2 This will depend upon whether $\Delta$ becomes zero or merely reaches a minimum value and then increases again. On the instrument $\Delta$ may be controlled, and the fringes made to be practically straight lines. 
tangent screw, chromatic fringes quickly appear. So distinct are they that the diffused light of the room is sufficient to give them very clearly.

Case 5. $\Delta=2 P \tan \varphi$ or $\Delta=2 t_{0} \tan \varphi$.

In this case $e=\mathrm{I}$.

Putting this value in equation (I 3 ) we obtain

$$
\mathrm{I}=\sin i \cos i \sqrt{\mathrm{I}+\tan ^{2} i+\tan ^{2},}
$$

which may be reduced to the form

$$
\sin \theta=\frac{\mathrm{I}}{\sqrt{\mathrm{I}+\sin ^{2} i}} .
$$

The limiting values of $\theta$ and $i$ are

$$
i= \pm 90^{\circ} \text {, and } \theta=90^{\circ} \pm 45^{\circ} \text {. }
$$

It is thus evident that the parabola cannot be seen unless $\theta>45^{\circ}$; and it is equally evident that $\theta$ cannot have this value unless the focal plane is quite close to $M_{1}$. This explains why the parabola is only seen for small values of $t_{0}$; or large values of $\varphi$, giving small values for $P$.

Case 6. $\Delta<2 P \tan \varphi$ or $\Delta<2 t_{0} \tan i$.

Then $e>\mathrm{I}$, and the curves are hyperbolæ. What has been said under Case 5 , as to the value of $t_{0}$ and $\varphi$ holds equally for this case, showing that the hyperbolic curves are only observed when the value of $t_{0}$ is very small.

Case 7. The Equilateral Hyperbola.

In this case $e=\sqrt{2}$ and equation (I4) reduces to

$$
\tan \theta=\sqrt{\frac{2}{\sin ^{2} i}+\sin ^{2} i-\mathrm{I}} .
$$

The following table based upon equation (I 8) shows the relation of $i$ and $\theta$.

If $i$ and $\theta$ be interchanged the conjugate system of hyperbolæ is obtained. In the general case of the hyperbola the minimum value of $\theta$ was found to be $45^{\circ}$. In the equilateral hyperbola the minimum value is $54^{\circ}$, showing that in this case the range of $t_{0}$ is still more restricted than in the general case. 
TABLE I.

\begin{tabular}{r|c|c|c|c}
\hline $\sin i$ & $i$ & $\tan \theta$ & $\theta$ & $P$ \\
\hline 0 & $0^{\circ}$ & $\infty$ & $90^{\circ}$ & $\frac{t_{0}}{\tan \phi} \times 0=0$ \\
0.5 & $30^{\circ}$ & 2.7 & $70^{\circ}$ & $\frac{t_{0}}{\tan \phi} \times 0.58$ \\
0.707 & $45^{\circ}$ & 1.87 & $62^{\circ}$ & $\frac{t_{0}}{\tan \phi} \times 1$ \\
0.877 & $60^{\circ}$ & 1.58 & $58^{\circ}$ & $\frac{t_{0}}{\tan \phi} \times 1.73$ \\
1 & $90^{\circ}$ & 1.4 & $54^{\circ} 40^{\prime}$ & $\frac{t_{0}}{\tan \phi} \infty=\infty$ \\
\hline
\end{tabular}

Case 7. Rotation of the Compensator. It is very instructive, for a given setting of the mirror $M_{2}$, to slowly rotate the compensating plate (C, Fig. I). The effect of such rotation is to change the length of path through the glass, and hence to alter the value of $t_{0}$. If the value of $t_{0}$ be already large, this change will not appreciably effect either the value of $P$ (Eq. 2) or that of $\Delta$ (Eq. I), and hence the value of $e$ remains sensibly unaltered.

If, however, $t_{0}$ be small the rotation of the compensator will produce a large relative change in $t_{0}$ and hence a large change in both $\Delta$ and $P$, resulting in a rapid change in the value of $e$.

For example, if the value of $t_{0}$ be small, and the compensating plate be parallel to plate $A$; then on rotation such as to diminish the optical path through the glass, the circular fringes are seen to change to the elliptical form, the value of $e$ becoming greater than zero.

As $t_{0}$ becomes still smaller, due to further rotation of the compensator, $e$ increases in value, and, corresponding to this the fringes pass through the Parabolic, into the hyperbolic form. Soon the conjugate system of hyperbolæ appear in the field of view, presenting a very pretty sight. If the compensator be rotated until it becomes parallel to $M_{1}$, the behavior of the fringes changes. As the parallel position is passed the direction of motion of the fringes reverses, and the value of $t_{0}$ begins to increase. The reversal in the movement of the fringes furnishes a very sensitive test for the parallelism of plates $C$ and $M_{1}$ (Fig. I). This test for parallelism 
might, from its sensitiveness, be made of more than passing value in other lines of work.

Case 8. $\varphi=0 ; t_{0}=0$. Under these conditions $P$ is indeterminate (Eq. 2). Therefore $\Delta$ is indeterminate (Eq. I), and so also $e$ by equations (I I) or (I2). If $t_{0}=0$ it is apparent that $M_{1}$ and $M_{2}$ coincide, and, as has already been pointed out, there should be no interference and hence no interference pattern.

This however will depend upon whether the two beams of light are exactly in phase. Now since one beam suffers internal and the other external reflection at plate $A$, if a difference of phase other than $\frac{1}{2} \lambda$ takes place interference would result and an interference pattern would be seen. Since $M_{1}$ and $M_{2}$ coincide the values of $i$ and $\theta$ would seem to be $0^{\circ}$, and the value of $e$ by equation (I3) would then be

$$
\begin{aligned}
& e=\sin i \cos i \sqrt{\mathrm{I}+\tan ^{2} i+\tan \theta} \\
& e=0
\end{aligned}
$$

and hence the fringes would be circles. Or we may consider that a difference of phase between the two beams, due to any cause, would be equivalent to a finite value for $t_{0}$. Then since $\varphi=0$, $e$ would be zero by equation (I I).

In the case of white light, in addition to the difference of phase due to reflection, the compensating plate $C$ introduces a dispersion ${ }^{1}$ effect (excepting when $C$ is strictly parallel to $A$ ) thus (in general) adding to the phase difference.

The result is that very brilliant chromatic circular fringes are to be seen even when $\varphi=0$ and $t_{0}=0$. The presence of fringes furnishes a test for the presence of a phase difference produced by reflection. Under these conditions as many as 147 circular chromatic fringes have been counted.

Equation (I 3), perhaps, sums up all of the preceding cases in as concise a manner as is possible. When $t_{0}$ is large it is evident that $i$ and $\theta$ must both be very small and hence $e=0$ (practically). As $t_{0}$ becomes smaller the possible values of $i$ and $\theta$ are larger giving the successively larger values for $e$.

${ }^{1}$ This is the "chromatism" investigated by Conn, C. R. t, xciii, I88I and by Michelson, Phil. May [5] I3, 236, I882. 
It is fascinating to observe the transformation of the interference fringes as they pass from one form of curve to another. Not only are the phenomena very beautiful in themselves but there is no better way of becoming thoroughly conversant with this admirable and many-sided instrument than is furnished by the study of the manifold curves presented by the interferometer. For the verification of the deductions given in this paper, I wish to thank Mr. A. C. Scott, of the staff of the Agricultural College, Kingston, R. I., who during the past summer collaborated with me in the physical laboratory of the University of Wisconsin.

Physical laboratory, Colorado College. 